\title{
LA INTIMIDAD TRANSGRESORA EN LA FICCIÓN DE COSTAMAGNA, FERNÁNDEZ, JEFTANOVIC, MATURANA Y MERUANE. ¿PODEMOS HABLAR DE UNA NUEVA GENERACIÓN LITERARIA?
}

\author{
Nan Zheng \\ The Graduate Center, CUNY, Nueva York, Estados Unidos \\ nzheng@gradcenter.cuny.edu
}

\section{RESUMEN / ABSTRACT}

El presente trabajo, enmarcado primordialmente en el discurso de la intimidad, los estudios sobre el régimen estético del arte de Jacques Rancière y las revisiones de Wendy Brown de las reflexiones de Foucault sobre el neoliberalismo, examina la ficción chilena de las últimas décadas, escrita por Alejandra Costamagna, Nona Fernández, Andrea Jeftanovic, Andrea Maturana y Lina Meruane. La producción literaria de estas autoras disiente de las normas patriarcales y de las establecidas por la racionalidad neoliberal dominada por el homo oeconomicus. En este trabajo se analizará la posibilidad de agrupar a las escritoras en una generación literaria de la postdictadura, lo que podría suponer más inconvenientes que ventajas, dado que constituiría un gesto en el que se podrían estar estableciendo particiones entre lo femenino y lo masculino, lo privado y lo público, lo sublime del arte y lo banal de la vida cotidiana.

Palabras Clave: Chile de la dictadura y la postdictadura, relatos de intimidad, neoliberalismo, generación literaria nueva.

\section{TRANSGRESSIVE INTIMACY IN THE FiCTION OF COSTAMAGNa, FERnándEZ, JEFTANOVIC, Maturana y MERUANE. CAN WE REFER to a NEW LITERARY GENERATION?}

The present work, framed primarily in the discourse of intimacy, Jacques Rancière's theory of the aesthetic regime of art and Wendy Brown's revision of Foucault's reflections on Neoliberalism, examines the Chilean fictions of the last two decades by Alejandra Costamagna, Nona Fernández, Andrea Jeftanovic, Andrea Maturana and Lina Meruane. The literary production of these female writers disagrees with patriarchal norms and those established by the neoliberal rationality dominated by the homo oeconomicus. This article analyzes the possibility of classifying these writers as a new literary generation of the post-dictatorship, 
which could be more inconvenient than beneficial, since it would represent a gesture which could be establishing divisions between feminine and masculine; private and public; the sublime in art and the banality of everyday life.

KEYWORDS: Chile under the dictatorship and during the post-dictatorship, tales of intimacy, neoliberalism, new literary generation.

Recepción: 29/04/2017

Aprobación: 10/07/2017

\section{¿EXISTE UNA NUEVA GENERACIÓN LITERARIA DE ESCRITORAS CHILENAS DE LA POSTDICTADURA?}

Después de su regreso a Chile en el año 1998, Roberto Bolaño expresó en "Fragmentos de un regreso al país natal" su apreciación sobre el valor literario de un grupo de escritoras chilenas, que nacieron entre finales de los sesenta y principios de los setenta, vivieron su niñez y adolescencia bajo el régimen militar pinochetista, y emprendieron su carrera literaria alrededor del fin del siglo. Bolaño escribió sobre ellas que estaban "armadas con todos elementos de la buena literatura" y "escriben como demonias", siendo una generación que "promete comérselo todo" (67-68) ${ }^{1}$. Hoy en día, casi veinte años después de que la figura canónica (y masculina) de Bolaño formulase este primer reconocimiento, las ficciones producidas por este grupo de escritoras, que incluye a Alejandra Costamagna, Nona Fernández, Andrea Jeftanovic, Andrea Maturana y Lina Meruane, son la prueba más contundente de su creatividad y originalidad ${ }^{2}$.

1 Invitado por la revista Paula como jurado del concurso de cuentos en 1998, Bolaño elaboró textos sobre las experiencias e impresiones de su retorno después de 25 años de ausencia y, en febrero del año siguiente, publicó "Fragmentos de un regreso al país natal" en la misma revista. El texto fue luego recopilado en la colección de crónicas Entre paréntesis: ensayos, artículos y discursos (1998-2003).

Las cinco escritoras gozan de un reconocimiento creciente tanto a nivel nacional como internacional. Han obtenido varios premios prominentes en el campo literario: Costamagna ganó el Premio Juegos Literarios Gabriela Mistral en 1996 con la novela En voz baja; Jeftanovic obtuvo el mismo premio en 2000 con Escenario de guerra; Fernández fue galardonada con el Premio Municipal de Literatura de Santiago 2003 por su primera novela Mapocho; a Maturana, el Consejo Nacional de la Cultura y las Artes de Chile le otorgó el premio del mejor libro de cuentos publicado en el año 2006, por la colección de cuentos No decir; entre los logros de Meruane, se destacan el Premio Anna Seghers de 2011 en Alemania y el XX Premio Sor Juana Inés de la Cruz en México del año 2012. 
Desde la primera década del dos mil, se han propuesto abordajes académicos que, con el propósito de agrupar y colocar una etiqueta generacional de la postdictadura, exploran los elementos temáticos y estilísticos comunes que configuran el panorama literario y artístico chileno reciente, al cual pertenecen las cinco escritoras. Ana Ros, en The Post-Dictatorship Generation in Argentina, Chile, and Uruguay: Collective Memory and Cultural Production, escribe sobre una generación de la postdictadura cuyos miembros se ven afectados, sin excepción, por la situación política de los regímenes militares del Cono Sur, y que se empeñan en articular, a través de la escritura, el arte y el cine, los fragmentos de las experiencias traumáticas desde la perspectiva de los hijos de padres activistas y de los bystanders, "independientemente de su nivel de comprensión de los acontecimientos actuales, e independientemente de la relación de su familia con la política" (4; traducción propia) ${ }^{3}$. Asimismo, existe un creciente número de entrevistas a las autoras, al igual que reportajes, que las presentan, junto con otros jóvenes escritores coetáneos, como "una destacada generación literaria... (que) (c)omparten una reconstrucción de la memoria entre lo íntimo y lo político", denominada como "los niños de la represión" (Querol).

Las propias escritoras también han expresado respectivamente opiniones variadas sobre la posibilidad de ser categorizadas como una generación literaria emergente de escritores criados durante la dictadura. Fernández, frente a la pregunta de cómo describiría la generación a la que ella pertenece, contestó "la generación medio guacha", que es una generación que tiene "conciencia de lo que pasaba, pero sin ser protagonistas porque los protagonistas fueron nuestros padres" (Fernández). Al ser entrevistada, Maturana expresó que, para su generación con una juventud "que quedó como algo «jodida»", el tema sobre la dictadura es inevitable en comparación con la gente más joven, y que las sensaciones o traumas infligidos durante tal época no cambian simplemente por la modernización del espacio urbano santiaguino (Maturana). La respuesta ofrecida por Costamagna coincide en cierto modo con lo que propuso Ilse Logie para el caso de la literatura argentina con la elaboración del término "los hijos simbólicos", dado que la escritora comentó que al decir la palabra "hijos" ella se refiere a "niñas y niños que no necesariamente tuvieron padres militantes ni comprometidos con las causas de la izquierda, pero que hoy, en su

Cita original: “...regardless of their degree of understanding of current events, and regardless of their family's relation to politics". 
adultez,... respiran las resacas del golpe" (Costamagna) ${ }^{4}$. Acerca de la etiqueta "los hijos de la dictadura", Meruane mantiene una opinión diferente. En una entrevista explicó que con este intento de nombrar una nueva generación de los hijos, se corre el riesgo de imponer un aislamiento generacional "como si (ellos) hubi(eran) sido parte", respecto a la literatura de la posmemoria como "relatos de segunda mano donde los narradores se hacen cargo como pueden de lo que vieron a medias o intuyeron" (cit. en Querol).

Además, los intentos de definir una nueva generación de narradoras, en algunos casos, conducen a un abordaje generalizador que conlleva el riesgo de descuidar ciertos factores importantes que podrían ser decisivos para el análisis de cada una de las narradoras y ficciones específicas. En la misma entrevista mencionada, Costamagna, al hablar sobre las obras de los hijos simbólicos, opinó:

[...] las maneras de procesar esas experiencias y esos hitos históricos comunes son muy diversas. Porque las biografías de cada cual, las vidas puertas adentro, difieren. Cada uno de esos libros surge de experiencias y sensibilidades singulares dentro de esa macro experiencia común... Son marcas históricas que cada uno procesa a su modo... Eso genera distintos matices y una heterogeneidad de miradas que me parece muy saludable (Costamagna).

Por tanto, cabría preguntarse si es posible hablar de una nueva generación literaria de escritoras chilenas de la postdictadura. Es indispensable, antes de nada, volver a revisar algunos de los métodos generacionales que se han aplicado en las investigaciones literarias, con el motivo de establecer el marco dentro del cual se efectuará el análisis sobre el caso de las cinco escritoras en este artículo. Los conceptos de generación sirven constantemente como uno de los instrumentos críticos predominantes de la taxonomía, con el objetivo de definir o autodefinir a escritores que, en la mayoría de los casos, no solo nacen y crecen en la misma era, sino que también están conectados por un interés común y por discursos similares. Especialmente durante los tiempos de crisis y la fase de recuperación posterior, frecuentemente se plantea la noción de

$4 \quad$ Ilse Logie, en su investigación sobre escritores argentinos que pertenecen a "la generación de los hijos", indica que esta generación argentina debe abarcar no solo a los hijos de padres desaparecidos, sino también a "todos los hijos simbólicos cuya infancia o adolescencia estuvo marcada por la experiencia dictatorial" (75-76). 
generación a grupos crecientes de escritores en cuyas literaturizaciones de la realidad se pueden percibir, en mayor o menor medida, actitudes inquisitivas y desafiantes con el statu quo tanto sociopolítico como estético. Al combinar la taxonomía generacional con el reconocimiento de la historicidad, Julius Petersen propuso, en Filosofía de la ciencia literaria, que la formación de nuevas generaciones es el "resultado de las luchas entre edades diferentes, entre una juventud que va madurando y haciéndose vieja y un espíritu juvenil que irrumpe pujante" (138). Para Pierre Bourdieu, los intentos de marcar fronteras entre distintas generaciones en el campo artístico se involucran en competiciones por la consagración y la legitimación. Escribe Bourdieu en Las reglas del arte:

Las diferencias en función del nivel de consagración separan de hecho a generaciones artísticas, definidas por el intervalo, a menudo muy corto, apenas de unos años a veces, entre unos estilos y unos estilos de vida que se oponen como lo "nuevo" y lo "viejo", lo original y lo "superado", dicotomías decisivas, a menudo vacías, pero suficientes para clasificar y hacer que existan, al menor coste, grupos designados -mejor que definidos- por etiquetas que responden al propósito de producir las diferencias que pretenden enunciar (188; cursivas del autor).

Sin embargo, se mantienen las interacciones dinámicas entre las cinco escritoras con las figuras literarias que las anteceden, y sería imposible confinarlos a todos a fenómenos literarios autosuficientes y apartados, así como trazar una línea rígida entre lo nuevo y lo viejo. La formación estética, que las cinco escritoras adquirieron en los talleres literarios encabezados por Diamela Eltit, Pía Barros y Antonio Skármeta desde los últimos años de la dictadura ha sido muy importante en su desarrollo. En los talleres de Eltit, Jeftanovic y Meruane publicaron sus primeras obras. Estas autoras estaban unidas, junto con otras escritoras jóvenes de aquel entonces, por "preocupaciones como la exploración de la memoria, ciertos efectos teatrales y una atención a la vida psíquica" (Kirkpatrick 63). Costamagna y Maturana se conocieron en el proyecto de talleres literarios para escritores jóvenes Ergo Sum, presididos por Pía Barros ${ }^{5}$. Maturana, siguiendo los pasos de la Generación de los 80, 
empezó a escribir ficciones eróticas (Cardone 273). Se trata de un erotismo íntimo (como por ejemplo en el cuento "Maletas" en (Des) encuentros (des) esperados), a veces incestuoso y/o perverso, como por ejemplo en la novela El daño.

Por otro lado, la exploración de un pasado fragmentado, a través de la mirada atenta de las memorias personales, no se limita a las obras literarias producidas por mujeres, sino que también se refina en varias ficciones escritas por escritores coetáneos como Alejandro Zambra (Formas de volver a casa) y Diego Zúñiga (Camanchaca), cuyos hijos-protagonistas, por medio de una narración de historias filiales en el presente, emprenden un proceso de reevaluación del pasado dictatorial.

\section{EL POTENCIAL TRANSGRESOR DE LOS RELATOS ÍNTIMOS}

Uno de los enfoques frecuentes en los estudios existentes sobre la producción literaria de las cinco escritoras consiste en los contrapuntos recuerdo-olvido, articulación-silencio y confesión-ocultamiento de la verdad, intensificados a lo largo del intrincado proceso de la construcción del pasado, así como de la construcción de la subjetividad. Tales contrapuntos destacan en El daño de Maturana y En voz baja de Costamagna, sobre niños o jóvenes atormentados por ser incapaces de entender lo que le pasó a su familia durante los años de la dictadura militar. En En voz baja destaca como una desmesura de desarticulación que satura el ambiente doméstico, ya que los susurros y los actos de callar a los hijos por parte de los adultos suceden de forma paralela a las circunstancias políticas del Chile bajo la dictadura, caracterizadas por la imposición del silencio, que obstaculiza la búsqueda de la verdad sobre la desaparición de su padre, que realiza Amanda. El dolor para memorizar de Elisa en El daño se vuelve obcecado mientras se entrecruzan las escenas, en presente, del viaje por el desierto con su compañera, y los recuerdos recurrentes sobre una infancia torturada.

El esfuerzo de las escritoras por contar historias íntimas de la familia, presentando sus momentos de quiebras y fallas, según Sergio Rojas, sirve como "el médium de su autoconciencia" (239). Lorena Amaro, quien investiga 
extensamente la imbricación entre la filiación y la ciudadanía en la narrativa de las autoras, propone que hay una doble construcción en la búsqueda de la herencia al explorar los momentos íntimos. Por un lado, buscan y cuestionan la herencia familiar para interrogar al sujeto en sí y el silencio de los padres; por el otro, "entrañan también interrogantes sobre la herencia literaria", que "cuestionan... no sólo la autoridad paterna, su verdad y su decir, sino que trascienden esa posibilidad hacia formas de crítica de la herencia social, cultural y política nada desdeñables" (Amaro, "Formas de salir de casa" 111). Además, en su presentación de las actas de la conferencia Estéticas de la intimidad, y siguiendo al filósofo español José Luis Pardo, Amaro hace un énfasis especial en la necesidad de investigar "el carácter político del tramado subjetivo... y la intimidad del dolor" en el ámbito latinoamericano desde los años ochenta (Amaro, "Presentación" 11). La crítica, indica que el poder transgresor de la forma discursiva del testimonio, que "atraviesa distintos tipos de texto" incluyendo el género de la novela, plantea posibilidades de "alteridad y resistencia" (11).

La dinámica de las relaciones familiares durante el Chile de la dictadura y la postdictadura, presentada por las ficciones de las cinco escritoras, ensambla una memoria colectiva caracterizada por una referencialidad histórica vigorosa, en vez de apoyarse en interpretaciones monolíticas. Por otro lado, los relatos desafían explícitamente al consenso patriarcal sobre los valores familiares y patrios, impuestos por los mecanismos represores del régimen militar y consagrados por el mesianismo militar, a través de una problematización de las relaciones paterno-filiales, con ejemplos como Cercada de Meruane y Escenario de guerra de Jeftanovic. Estos relatos íntimos desafían los discursos patriarcales que propagan que "la solidaridad familiar sea el primer fundamento del verdadero patriotismo" (Valores patrios 11) y que la misión familiar sea "un valioso patrimonio de normas, de conducta moral y de principios" (34). En el prólogo de Hablan los hijos: discursos y estéticas de la perspectiva infantil en la literatura contemporánea, Jeftanovic articula que la narrativa, con el artificio de simular las perspectivas de niños para "denunicia(r) la historia, las injusticias, el autoritarismo, las desviaciones del mercado y más problemáticas sociales y existencialistas..., es siempre una trampa", que "pasa a ser una máquina con función creadora, que despliega procesos de subjetivación y empuja el lenguaje y el imaginario a límites y zonas insospechadas" (13).

El potencial transgresor de los relatos filiales íntimos entre las esferas público-privada y cotidiano-política, tiene consonancia con lo que Josefina 
Ludmer propuso en "Tretas del débil". Es decir, que se debería "aceptar la esfera privada como campo propio de la palabra de la mujer... (y) desde el lugar asignado y aceptado, se cambia no sólo el sentido de ese lugar sino el sentido mismo de lo que se instaura en él" (53). Entre los mecanismos de los relatos de las cinco escritoras chilenas, que desafían al régimen social patriarcal, están el cuento "Agujas del reloj" de Costamagna, donde se abordan temas eróticos y tabúes sexuales, con ejemplos como la relación incestuosa padrehija; Mapocho de Fernández, donde la autora "invierte la noción de romance nacional" con los temas de violación, incesto y orfandad (particularmente en las escenas donde el Indio codicia el cuerpo de su hermana, la Rubia), que "constituyen una alegoría del fracaso de un proyecto nacional fundado en el terror a la diferencia del otro" (Opazo 43-44; cursiva del autor); y "Hojas de afeitar" de Meruane, un cuento sobre un grupo de estudiantes adolescentes de un colegio privado y su ritual de afeitado a escondidas, donde afloran descripciones eróticas sobre las partes del cuerpo femenino.

Por medio de la elaboración de historias familiares "anormales" y "perversas", en varias obras de estas escritoras se ven registros del disenso que se resisten a la idea de competencia que infiltra la racionalidad neoliberal en la mentalidad individual y social. Los discursos y prácticas de normalización y normativización, promovidos por tal racionalidad durante los años de la dictadura y la postdictadura, no solo han venido transformando las esferas públicas de la sociedad chilena, sino que también infiltran y erosionan de manera sigilosa los espacios privados de la vida familiar. A este respecto, en el libro Undoing the Demos: Neoliberalism's Stealth Revolution, Wendy Brown afirma que, al subvencionar el declive del homo politicus democrático, la lógica del mercado neoliberal construye y populariza la imagen del homo ceconomicus, que es una falsa personificación de la libertad e igualdad y de las ideologías conservadoras sobre la familia: "Todas las conductas son conductas económicas; todas las esferas de la existencia están enmarcadas y medidas por los términos y métricas económicos, e incluso cuando tales esferas no son directamente monetizadas ... somos sólo, y en todas partes, homo æeconomicus" (10; traducción propia) ${ }^{6}$. Los estudios de Brown abren

$6 \quad$ Cita original de Brown: "All conduct is economic conduct; all spheres of existence are framed and measured by economic terms and metrics, even when those spheres are not directly monetized ... we are only and everywhere homo oeconomicus". Las oportunas revisiones y actualizaciones teóricas propuestas por Brown sobre lo que formuló Michel Foucault sobre la racionalidad neoliberal en la serie de seminarios dictados en el Collège 
nuevos horizontes a la convocatoria de esfuerzos para salir fuera de los marcos preestablecidos por la lógica de la gobernanza neoliberal, para distinguir las falsas promesas bajo el disfraz de la libertad, y para explorar los mecanismos posibles (en el caso de este trabajo, los relatos íntimos escritos por mujeres) en la defensa de los valores fundamentales de la democracia. Brown expone que, de manera sigilosa, la dominación hegemónica de la racionalidad neoliberal ataca a los elementos básicos de la democracia, incluyendo su vocabulario, principios de justicia, culturas políticas y sobre todas las cosas, los imaginarios democráticos (17). Reduce las nociones de la libertad y la igualdad en "el derecho a la crueldad empresarial ... (que) dio paso a mundos ubicuamente competitivos de ganadores y perdedores" (Brown, "Sacrificial Citizenship" 3; traducción propia) ${ }^{7}$. De acuerdo con la imagen específica y única de lo económico, la racionalidad neoliberal desintegra la ciudadanía democrática -el homo politicus-(10), y produce al homo œeconomicus desdemocratizador y hegemónico, efecto de "la maximización de la competencia ... y una serie de políticas monetarias y sociales favorables a las empresas, e indiferentes ante la pobreza, el desarraigo social, la aniquilación cultural, el agotamiento de los recursos a largo plazo y la destrucción del medio ambiente" (Brown, "Neoliberalism" 38; traducción propia) ${ }^{8}$.

Asimismo, los estudios de Brown sobre la imbricación entre el neoliberalismo y otras racionalidades desdemocratizadoras en los Estados Unidos, ofrecen

de France entre 1978 y 1979, publicada con el título de The Birth of Biopolitics, resultan interesantes para la investigación sobre el Chile neoliberal en varios sentidos. En sus cursos, Foucault interpretó el neoliberalismo como una racionalidad gubernamental emergente que marca un punto de inflexión en la evolución histórica de las filosofías políticas liberales sobre el rol del gobierno frente al mercado (Flew 44). Foucault ofreció una presentación detallada sobre la genealogía del homo æeconomicus y su definición del homo æeconomicus neoliberal como sujeto de interés en el seminario número 11, del 28 de marzo, 1979, en The Birth of Biopolitics. Para él, el homo oecomomicus neoliberal, caracterizado por la competencia e individualización, era un empresario de sí mismo (226), un "sujeto de interés ... cuya acción tiene, a través de la intensificación del interés, un valor multiplicador y beneficioso" (276; traducción propia). En pocas palabras, los comentarios de Foucault sobre el neoliberalismo en sus seminarios indicaban una actitud no crítica (Flew 59). Por su parte, Brown lo considera en términos puramente negativos, normativos y desdemocratizadores.

Cita orignal: “...the right to entrepreneurial ruthlessness ... (that) gave way to ubiquitously competitive worlds of winners and losers".

Cita original: “...maximised competition ... and a range of monetary and social policies favorable to business and indifferent toward poverty, social deracination, cultural decimation, long term resource depletion and environmental destruction". 
una perspectiva valiosa para investigar los tres lados del triángulo del poder en el Chile de la dictadura y la postdictadura: el neoliberalismo, el autoritarismo y el mesianismo militar. En el artículo titulado "American Nightmare: Neoliberalism, Neoconservatism, and De-Democratization", Brown explora en profundidad la imbricación entre el neoliberalismo y el neoconservadurismo. Propone que ambas ideologías, a pesar de sus divergencias y con el apoyo del cristianismo fundamentalista, convergen en producir sujetos indiferentes hacia los derechos sustantivos de la ciudadanía democrática (690). Además, la idea del sacrificio hace disminuir la brecha o paradoja entre el neoliberalismo y el neoconservadurismo. En "Sacrificial Citizenship: Neoliberalism, Human Capital, and Austerity Politics", Brown sugiere que la racionalidad neoliberal comparte con algunas ideologías autoritarias (por ejemplo, el fascismo) la evaluación del productivismo y del espíritu de sacrificio "para conseguir un bien mayor en el que todos están integrados, sin que ninguno debiera esperar un beneficio personal" (12; traducción propia) $)^{9}$.

En Av. 10 de julio Huamachuco de Fernández, destacan el sacrificio del sujeto neoliberal y la alerta contra el ascenso del homo oeconomicus al poder, investigados por Brown en dicho artículo. Los dos protagonistas de esta novela, Juan y Greta, intentan escapar de las falsas promesas de felicidad y libertad económicas que promete la lógica del mercado neoliberal. Una lógica que contribuye a la amnesia sobre el pasado de la nación, y programa a su gusto la construcción de una ciudadanía feliz, o mejor dicho, consumidores felices. A ambos protagonistas les es obstaculizado el derecho a vivir con dignidad, por no querer obedecer las rutinas normalizadas por tal racionalidad ni olvidar la violencia de sus pasados. Son aislados, expulsados, wretched, como escribe Sara Ahmed en el artículo "Killing Joy: Feminism and the History of Happiness", donde la autora reflexiona sobre la necesidad de investigar las vidas y sufrimientos de los alborotadores, desgraciados, extraños, disidentes, joy-killers, así como de elaborar un archivo de lo infeliz, donde se registren las distintas luchas particulares (573): como las de Juan y Greta, en contra de una noción de la felicidad generalizada y prefigurada por la lógica de la normatividad neoliberal.

9 Cita original: “...for a greater good into which all are integrated but from which none should expect personal benefit”. 


\section{LO SENSIBLE REDISTRIBUIDO Y LO INTIMO SUBVERSIVO}

Los relatos íntimos escritos por estas autoras chilenas demuestran el potencial democratizador para renegociar y desestabilizar las fronteras entre diferentes divisiones de lo sensible (entre lo público y lo privado, lo político y lo estético, y lo femenino y lo masculino), y superan las limitaciones de los métodos generacionales. Asimismo, es indispensable tener presente el potencial transgresor de los relatos íntimos de las escritoras chilenas entre el arte y la vida. En estas obras, las resistencias cotidianas suponen un poder emancipador e igualitario, ya que, como indicó Jacques Rancière en El desacuerdo: política y filosofía, ofrecen nuevas oportunidades para renegociar las posibilidades de vidas alternativas, mediante una revisión de los síntomas de la Historia con mayúscula, caracterizada por una amnesia colectiva en los años de la postdictadura. Rancière destaca la capacidad de la política para discernir el potencial emancipador de las luchas y situaciones adversas más cotidianas e insignificantes (Tanke 63), para luego convertirlas en medios eficaces para el alcance de la igualdad y la democracia. En El desacuerdo afirma:

Lo que constituye el carácter político de una acción no es su objeto o el lugar donde se ejerce sino únicamente su forma, la que inscribe la verificación de la igualdad en la institución de un litigio, de una comunidad que sólo existe por la división (47).

La igualdad entre el dominante y el dominado, la indiferencia hacia la localización específica de los escenarios de resistencia (sea público o privado), la eliminación de particiones entre disciplinas y discursos, son los puntos de partida de la elaboración de Rancière, en El malestar en la estética, de sus teorías sobre la redistribución de lo sensible por el régimen estético de la literatura y el arte, y la paradoja inherente de este régimen. Hay dos determinantes que establecen esta paradoja metapolítica fundamental: la heteronomía y la autonomía de la literatura y el arte. Rancière explica:

La política del arte dentro del régimen estético del arte, o más bien su metapolítica, se encuentra determinada por esta paradoja fundadora: en este régimen, el arte es arte en la medida en que es también no arte, una cosa distinta que el arte ... Existe una contradicción originaria y que actúa de manera incesante. La soledad de la obra lleva consigo una promesa de emancipación. Pero el cumplimiento de la promesa implica la supresión del arte como realidad separada, su transformación en una forma de vida (Rancière, El malestar 48-49). 
$\mathrm{El}$ arte por el arte, el arte por la vida. La literatura que respeta esta relación paradójica entre su autonomía y su heteronomía es capaz de borrar el límite entre el arte y la vida sin perder su identidad esencialmente estética, y de realizar la promesa de vidas alternativas, caracterizada por la igualdad y la democracia, manteniendo la distancia con la realidad social (Tanke 85). Las ficciones de las cinco escritoras sobre las intervenciones modestas y cotidianas, en el espacio íntimo y privado, imparten proposiciones relativas a la emancipación humana, mientras mantienen el carácter distintivo del arte: el arte de escribir.

Los pormenores de resistirse en el espacio íntimo de la vida humana y su potencia subversiva desafían al orden del consenso. Lo argumenta Nora Catelli, en la introducción de En la era de la intimidad, afirmando que el espacio íntimo es "una herramienta para la comprensión de las transformaciones históricas" (10), y diciendo que lo indirecto de las flexiones sobre la condición sociopolítica, lo ambiguo entre los escenarios público y privado y entre las dimensiones imaginaria y real, confieren a la narrativa de lo íntimo el potencial peligroso y dinamizador de una "vivencia histórica" (10). Lauren Berlant propone que las relaciones discursivas modernas de la intimidad crean espacios nuevos por medio de la usurpación de lugares o espacios destinados a otros tipos de relación, e incluso, relaciones no propias ("Intimacy" 282), lo que está en consonancia con la redistribución rancieriana de lo sensible. El discurso de lo público íntimo de la femineidad, abordado por Berlant en The Female Complaint: The Unfinished Business of Sentimentality in American Culture, desestabiliza las fronteras entre lo privado y lo público. El carácter ambivalente y yuxtapolítico de lo público íntimo genera un modelo estético nuevo: "an aesthetic of attachment", o una estética de apego/cariño que no se limita a las formas y sentimientos convencionales ni definitivos ("Intimacy" 285). Berlant, siguiendo el estudio de Deleuze y Guattari sobre Kafka y la literatura menor, formula que el discurso de la intimidad menor de los grupos minoritarios, con el ejemplo de la narrativa esclavista estadounidense desde el siglo XIX, "se ha visto obligado a desarrollar una estética de lo extremo, con el motivo de empujar estos espacios a emerger, a través de gestos tanto pequeños como grandes" (285; traducción propia) ${ }^{10}$.

10 Cita original: "ha(s) been forced to develop aesthetics of the extreme to push these spaces into being by way of small and grand gestures". Véase Queen of America Goes to Washington City: Essays on Sex and Citizenship, sobre la circulación de la narrativa de la 


\section{CONCLUSIÓN}

En el artículo titulado "La 'generación' como ideología cultural” sobre el caso de la literatura mexicana, Ignacio Sánchez Prado plantea que "el análisis estético e histórico de las generaciones jóvenes implica un grado de densidades que exceden por mucho el poder explicativo de la generación" (18-19). Este también es el caso de la producción estética de las cinco escritoras chilenas en el presente trabajo. Aislarlas en una generación literaria, imponiendo etiquetas de mujer / postdictadura / nueva, podría suponer más inconvenientes que ventajas, porque no es deseable reducir esta generación a una categoría estética autoevidente, que consista en un gesto por el que se establezcan particiones entre lo femenino y lo masculino, lo privado y lo público, y lo sublime del arte y lo banal de la vida cotidiana.

\section{BIBLIOGRAFÍA}

Ahmed, Sara. "Killing Joy: Feminism and the History of Happiness". Signs 35/3 (2010): 571-594. Amaro, Lorena. "Presentación". Estéticas de la intimidad. Ed. Lorena Amaro et al. Santiago: Pontificia Universidad Católica de Chile, 2009. 9-13.

"Formas de salir de casa, o cómo escapar del Ogro: relatos de filiación en la literatura chilena reciente". Literatura y Lingüistica 29 (2013): 109-129.

Berlant, Lauren G. "Intimacy: A Special Issue”. Intimacy, número especial de Critical Inquiry 24/2 (1998): 281-288.

Queen of America Goes to Washington City: Essays on Sex and Citizenship. Durham: Duke University Press Books, 2012.

The Female Complaint: The Unfinished Business of Sentimentality in American Culture. Durham: Duke University Press, 2008.

Bolaño, Roberto. "Fragmentos de un regreso al país natal". Entre paréntesis: Ensayos, artículos y discursos (1998-2003). Ed. Ignacio Echeverría. Barcelona: Anagrama, 2013. 59-70.

Bourdieu, Pierre. Las reglas del arte. Génesis y estructura del campo literario. Trad. Thomas Kauf. Barcelona: Anagrama, 1995.

Brown, Wendy. "American Nightmare: Neoliberalism, Neoconservatism, and De-Democratization." Political Theory 34/6 (2006): 690-714.

intimidad menor en el espacio público, que convierte las preocupaciones privadas y corporales en contra-discursos a la estructura dominante del poder. 
"Neoliberalism and the End of Liberal Democracy". Edgework: Critical Essays on Knowledge and Politics. New Jersey: Princeton University Press, 2005. 37-59.

"Sacrificial Citizenship: Neoliberalism, Human Capital, and Austerity Politics." Constellations 23/1 (2016): 3-14.

Undoing the Demos: Neoliberalism's Stealth Revolution. New York: Zone Books, 2015.

Cardone, Resha Sophia. “Acting up and Carrying on: Women Writers of Chile, 1945-2006”. Tesis doctoral. University of Kansas, 2006.

Catelli, Nora. "Introducción: en la era de la intimidad". En la era de la intimidad. Seguido de: El espacio autobiográfico. Rosario: Beatriz Viterbo Editora, 2007. 9-28.

Costamagna, Alejandra. "Había una vez una pájaro (o las voces de Alejandra Costamagna)". Entrevista por Lorena Amaro. 60 Watts. 2-septiembre 2013. Recuperado de: http://60watts. cl/2013/09/entrevista-alejandra-costamagna/

De Querol, Ricardo. “Los niños de la represión chilena llenan los silencios”. El País. 7-Julio 2015. Recuperado de: http://cultura.elpais.com/cultura/2015/06/09/babelia/1433843677_532023.html

Fernández, Nona. "Nona Fernández: vengo de una generación medio guacha”. Entrevista por Pedro Pablo Guerrero. El Mercurio. 12-agosto 2012. Recuperado de: http://diario. elmercurio.com/detalle/index.asp?id=\%7B8792e430-e2f4-45ee-b96b-2575147b4884\%7D

Flew, Terry. "Michel Foucault's The Birth of Biopolitics and Contemporary Neo-liberalism Debates". Thesis Eleven 108/1 (2012): 44-65.

Foucault, Michel. The Birth of Biopolitics: Lectures at the Collège de France 1978-79. Ed. Michel Senellart, trad. Graham Bruchell, New York: Palgrave Macmillan, 2008.

Jeftanovic, Andrea. "Prólogo". Hablan los hijos: discursos y estéticas de la perspectiva infantil en la literatura contemporánea. Santiago: Editorial Cuarto Propio, 2011. 1-19.

Kirkpatrick, Gwen. "El 'hambre de ciudad' de Diamela Elitit: forjando un lenguaje del Sur". Letras y proclamas: la estética literaria de Diamela Eltit. Ed. Bernardita Llanos M. Santiago: Editorial Cuarto Propio, 2006. 33-68.

Logie, Ilse. "Más allá del 'Paradigma de la memoria': la autoficción en la reciente producción postdictatorial Argentina. El caso de 76 (Félix Bruzzone)". Pasavento: Revista de Estudios Hispánicos 3/1 (2015): 75-89.

Ludmer, Josefina. "Tretas del débil". La sartén por el mango. Encuentro de escritoras latinoamericanas. Ed. Patricio Elena González y Eliana Ortega. Río Piedras: Huracán, 1985. 47-54.

Maturana, Andrea. "La palabra tiene algo de magia negra y blanca: entrevista a Andrea Maturana." Entrevista por Vania Barraza Toledo. Grafemas Boletín Electrónico de la AILCFH. Febrero 2007. Recuperado de: http://people.wku.edu/inma.pertusa/encuentros/ grafemas/febrero_07/vania.html

Opazo, Cristián. “Mapocho, de Nona Fernández: la inversión del romance nacional”. Revista Chilena de Literatura 64 (2004): 29-45.

Petersen, Julius. "Las generaciones literarias". Filosofia de la ciencia literaria. Trad. Carlos Silva. México: Fondo de Cultura Económica, 1946. 137-193.

Rancière, Jacques. El malestar en la estética. Trad. Miguel Petrecca et al. Buenos Aires: Capital Intelectual, 2011. 
El desacuerdo: politica y filosofía. Trad. Horacio Pons. Buenos Aires: Ediciones Nueva Visión, 1996.

Rojas, Sergio. "Profunda superficie: memoria de lo cotidiano en la literatura chilena". Revista Chilena de Literatura 89 (2015): 231-256.

Ros, Ana. The Post-Dictatorship Generation in Argentina, Chile, and Uruguay: Collective Memory and Cultural Production. New York: Palgrave Macmillan, 2012.

Sánchez Prado, Ignacio. “La 'generación' como ideología cultural: el Fonca y la institucionalización de la 'narrativa joven' en México”. Explicación de Textos Literarios 36/1-2 (2007): 8-20.

Secretaría de la Mujer de Chile. "Valores patrios y valores familiares", Cuadernos de Difusión 7, 1982.

Tanke, Joseph J. Jacques Rancière: An Introduction. Philosophy, Politics, Aesthetics. London: Continuum, 2011. 